\title{
2 Engineering Molecular Immunity against Plant Viruses
}

\author{
3 Syed Shan-e-Ali Zaidi ${ }^{1,2}$, Manal Tashkandi ${ }^{1}$, and Magdy M. Mahfouz ${ }^{1, *}$ \\ 4 \\ $5 \quad{ }^{1}$ Laboratory for Genome Engineering, Division of Biological Sciences, 4700 King Abdullah \\ 6 University of Science and Technology, Thuwal 23955-6900, Saudi Arabia \\ 7 \\ ${ }^{2}$ National Institute for Biotechnology and Genetic Engineering (NIBGE), Faisalabad, Pakistan \\ 10 *Correspondence: M.M. Mahfouz \\ 11 magdy.mahfouz@kaust.edu.sa
}

\section{Abstract}

13 Genomic engineering has been used to precisely alter eukaryotic genomes at the single-base

14 level for targeted gene editing, replacement, fusion, and mutagenesis, and plant viruses such as

15 Tobacco rattle virus have been developed into efficient vectors for delivering genome-

16 engineering reagents. In addition to altering the host genome, these methods can target pathogens

17 to engineer molecular immunity. Indeed, recent studies have shown that clustered regularly

18 interspaced short palindromic repeats (CRISPR)/CRISPR-associated 9 (Cas9) systems that target

19 the genomes of DNA viruses can interfere with viral activity and limit viral symptoms in planta,

20 demonstrating the utility of this system for engineering molecular immunity in plants.

21 CRISPR/Cas9 can efficiently target single and multiple viral infections and confer plant

22 immunity. Here, we discuss the use of site-specific nucleases to engineer molecular immunity

23 against DNA and RNA viruses in plants. We also explore how to address the potential challenges

24 encountered when producing plants with engineered resistance to single and mixed viral

25 infections. 


\section{Keywords}

27 Site-specific nuclease, genome engineering, CRISPR/Cas9, Geminiviruses, tobacco rattle virus

\section{1. Introduction}

The ability to alter DNA sequences in a site-specific manner and to generate gene

31 mutants and protein variants can facilitate the interrogation of gene function and understanding

32 of functional biology. The term "genome engineering" refers to the precise, site-specific

33 manipulation of the genome at the single-base level ${ }^{1}$. Genome engineering uses site-specific

34 nucleases (SSNs) to generate site-specific double-stranded breaks (DSBs) in the genome. DSBs

35 trigger the cellular repair machinery ${ }^{2}$ and two major DNA repair pathways, the imprecise non-

36 homologous end joining (NHEJ) pathway and the precise homology-directed repair (HDR)

37 pathway, can repair DSBs ${ }^{3}$ (Figure 1). NHEJ repair is suitable for site-specific mutagenesis and

38 for subsequent functional knockout of genes. Therefore, genome engineering via NHEJ is ideal

39 for interrogating gene functions. By contrast, HDR uses a DNA template to provide the

40 information needed by the HDR repair machinery to copy the sequence across the break ${ }^{4}$. HDR

41 constitutes a precise form of repair and is ideal for generating gene and protein variants based on

42 a DNA template that is supplied with the nuclease that produces the DSBs. Several genome

43 engineering platforms have been developed and repeatedly improved, but limitations to their

44 efficiency and ease of use remain, especially in plants ${ }^{5}$. One limitation is the choice of vector for

45 delivering genome-engineering reagents into the host cell. 
Work in a variety of systems has used viruses to deliver genome-engineering reagents

47 into target cells ${ }^{6}$. For example, in mammalian systems, the effective delivery of genome

48 engineering reagents has been achieved using viruses, including retroviruses ${ }^{7,8}$, adenoviruses ${ }^{9}$,

49 and adeno-associated viruses ${ }^{10}$. These viral vectors can be delivered into virtually any human

50 cell type ${ }^{8,10,11}$ and function as templates not only for expression of the designer site-specific

51 nucleases (SSNs), but also for targeted chromosomal integration of foreign DNA ${ }^{6,11}$. Recent

52 studies have developed DNA and RNA viruses for genome engineering in plants ${ }^{12,13}$.

53 In addition to using viruses as delivery vectors, genome engineering reagents can now be

54 used to target invading DNA viruses for degradation and to establish plant molecular immunity

55 against single and multiple viral infections ${ }^{14,15}$. For example, Ali et al. engineered tobacco plants

56 with clustered regularly interspaced short palindromic repeats (CRISPR)/CRISPR-associated 9

57 (Cas9) targeting the Tomato yellow leaf curl virus (TYLCV), establishing virus interference and

58 significantly limiting viral symptoms ${ }^{14}$. Similarly, Baltes et al. and Ji et al ${ }^{16,17}$ established virus

59 interference in tobacco against Bean yellow dwarf virus (BeYDV) and in Arabidopsis against

60 Beet severe curly top virus (BSCTV). Here, we discuss the current research on the use of genome

61 engineering to develop virus interference strategies, and address the potential challenges of

62 engineering plants with effective, durable virus resistance.

63

\section{$642 . \quad$ The use of SSNs for genome engineering}

65 SSNs, including zinc finger nucleases (ZFNs), transcription activator-like effector

66 nucleases (TALENs) ${ }^{18-23}$, and the recently developed CRISPR/Cas $9{ }^{24-26}$ system, have been used

67 to produce targeted mutations and edit the genomes of various eukaryotic species ${ }^{1}$ (Figure 1). 
68 ZFNs and TALENs have been used for targeted editing of plant genomes ${ }^{27-29}$, but the specificity

69 of these $\mathrm{SSNs}^{30}$ comes from protein-DNA binding and therefore ZFNs and TALENs require

70 protein engineering for each user-selected target sequence ${ }^{31}$. Engineering ZFNs and TALENs is

71 time-consuming and resource-intensive and must be performed by skilled molecular biologists ${ }^{32,}$

$72{ }^{33}$. Several repeat assembly protocols have been developed for TALEN engineering, but the need

73 for two TALEN monomers to bind to the sense and antisense strands simultaneously complicates

74 the broad utility and applicability of this technique in plants ${ }^{4}$. These limitations have been

75 largely overcome in the recently developed CRISPR/Cas9 system ${ }^{34-36}$. CRISPR/Cas9

76 engineering is highly predictable and much easier to perform than the other techniques, with

77 greater reproducibility and affordability ${ }^{37}$.

78 In nature, the CRISPR/Cas9 system provides adaptive molecular immunity to bacterial

79 and archaeal species to confer resistance against invading DNA species, including conjugative

80 plasmids and phages ${ }^{38}$. The CRISPR/Cas9 locus in the bacterial genome is typically composed

81 of Cas genes and the repeat and spacer sequences downstream of a leader sequence ${ }^{39,40}$.

82 CRISPR/Cas9- based immunity is achieved via three distinct phases: foreign DNA is acquired

83 from the plasmid or phage (acquisition phase), the CRISPR locus is transcribed and CRISPR

84 RNA processing and maturation take place (transcription and maturation phase), and the paired

85 trans-activating crRNAs (tracrRNAs) and CRISPR RNAs (crRNAs) are used as guides for the

86 Cas9 endonuclease to target or interfere with subsequent invasions (interference phase) ${ }^{39,41,42}$.

87 Therefore, the CRISPR/Cas9 system acquires pieces of DNA from an invading phage or plasmid

88 and uses these sequences as molecular records to guide Cas9 endonuclease to bind to the phage

89 or plasmid DNA in subsequent invasions, binding in a region preceding the protospacer

90 associated motif (PAM) sequence ${ }^{43}$. The PAM, a trinucleotide sequence that is important for 
91 Cas9 activity, is used to distinguish self from non-self DNA ${ }^{44}$. The PAM sequence of

92 Streptococcus pyogenes Cas9 is NGG (with N being any nucleotide) and that of S. thermophilus

93 Cas9 is $\mathrm{NGGNG}^{43,45}$.

94 Notably, the tracrRNAs and crRNAs required for Cas9 specificity can be fused to

95 generate a chimeric single-guide RNA molecule (sgRNA) that can direct Cas9 to a specific

96 sequence preceding the PAM trinucleotide sequence ${ }^{46}$. Since engineering via the CRISPR/Cas9

97 system involves changing only the 20 -nucleotide sequence, the CRISPR/Cas9 system is the

98 system of choice for genome engineering applications ${ }^{26}$.

The CRISPR/Cas9 system is based on Watson-Crick base pairing between the sgRNA

100 and the DNA of the target genome ${ }^{47}$. Therefore, the CRISPR/Cas 9 system supersedes and

101 outperforms other systems due to its ease of use, reproducibility, and affordability ${ }^{26,48}$. The

102 CRISPR/Cas9 system has recently been used for various genome-engineering applications across

103 eukaryotic species, including plants ${ }^{49-51}$. For example, the CRISPR/Cas9 system was used to edit

104 the wheat and maize genomes for targeted trait development ${ }^{52-54}$. Various CRISPR/Cas9-based

105 genome engineering modalities have been used involving different Cas9 variants ${ }^{55}$ or

106 catalytically inactive Cas9 (dCas9) to modulate gene expression patterns and to subsequently

107 interrogate gene functions ${ }^{56,57}$.

108 Editing of genes and genomes usually involves site-specific mutagenesis, including DNA

109 addition, deletion, or creation of gene fusions. For any genome-engineering platform or

110 modality, there are always concerns about specificity, delivery of reagents, and HDR efficiency

111 in the target cells. Therefore, ongoing, active research aims to improve the specificity, delivery,

112 and editing efficiency in a target cell or species to maximize the usefulness of the genome 
113 engineering modality ${ }^{38}$. Very recently, a novel class of DNA-guided SSNs involving FEN:FokI

114 has been reported; these are expected to be suitable for various engineering purposes ${ }^{58}$.

116 3. Viruses for delivery of genome engineering reagents

117 As mentioned in the Introduction, many studies in mammalian systems have successfully

118 used viruses to deliver genome engineering reagents. For plants, the TRV RNA virus has great

119 potential for use as a vector for genome engineering. Important features of the TRV-based

120 delivery system include the following: 1) TRV can systemically infect many plant species, both

121 naturally and under laboratory conditions; 2) TRV can easily introduced into plants via

122 Agrobacterium and delivered into the growing points of the plant; 3) the small genome size of

123 TRV facilitates cloning, multiplexing, library construction, and agroinfection; and 4) the RNA

124 genome of this virus does not integrate into the plant genome. A TRV-based expression system

125 has been developed for transient delivery of ZFNs into a variety of tissues and cells of intact

126 plants, representing a non-transgenic approach for ZFN delivery and mutant plant production ${ }^{59}$.

127 TRV has a bipartite genome, with two RNA components: RNA1 (or TRV1) and RNA2 (or

128 TRV2). In the above-mentioned system, RNA2 was replaced with RNA for the Zif268:FokI

129 ZFN, and targeted genome modifications were recovered in somatic tobacco (Nicotiana

130 tabacum) and petunia (Petunia hybrida) cells at an integrated reporter gene ${ }^{59}$. TRV systemically

131 infected its hosts, and ZFN-mediated, precise mutagenesis was clearly observed in newly

132 developed infected tissues, as measured by the activation of a mutated reporter transgene in

133 tobacco and petunia plants ${ }^{59}$. 
If TRV could infect germ cells, this would improve its utility as a vector for genome engineering, making it possible to harvest mutant seeds from infected plants. Indeed, the ability of TRV to move to developing buds and regenerating tissues has enabled researchers to recover

137 mutated plants ${ }^{59}$. Sequence analysis and examination of transmission of the mutations to the next 138 generation confirmed the stability of the ZFN-induced genetic changes ${ }^{59}$. Because TRV can 139 infect a wide range of plant species, it provides a viable alternative to the production of ZFN140 mediated mutants while avoiding the use of classical plant transformation methods.

141 Recently, we developed a TRV system that delivers single and multiple sgRNAs for 142 targeted editing of the host genome and for targeting invading DNA viruses ${ }^{12,14,15,60}$ (Figure 3).

143 In this system, TRV delivers single or multiple sgRNAs for genomic targets to modify the 144 genomes of host plants, including tobacco and Arabidopsis. To determine whether the newly 145 developed TRV could produce and deliver sgRNA molecules systemically and to evaluate the 146 persistence of the genomic modification in growing tissues, the engineered TRV was delivered 147 by agroinfiltration and reconstituted in the leaves of $N$. benthamiana expressing Cas9 ${ }^{12,60}$.

148 Interestingly, TRV-mediated delivery of sgRNA was not limited to the original infiltrated plants; 149 this precise targeting can also be transmitted to the next generation (Figure 2). This TRV150 mediated sgRNA delivery system stands out because it bypasses the requirement for plant 151 transformation and tissue culture ${ }^{12}$. This technique opens up new possibilities for producing 152 plants with desired traits using CRISPR/Cas9 without the need for laborious, time-consuming 153 intermediate steps ${ }^{12}$.

154 One potential limit of RNA viruses is that these viruses are limited in the amount of 155 heterologous DNA they can carry (approximately 2-3 kb); excess cargo results in the loss of 156 systemic movement or recombinational loss of the cargo DNA ${ }^{61,62}$. This limits the utility of 
157 these viruses for delivering TALENs or Cas9 expression cassettes, which typically exceed the

158 virus's cargo capacity. However, for relatively small molecules such as ZFNs, meganucleases,

159 and sgRNAs, TRV was proven to be an effective delivery agent, achieving mutagenesis in

160 somatic and germline cells of several plant species, including tobacco ${ }^{59}$, . $_{\text {benthamiana }}{ }^{12,60}$,

161 and petunia ${ }^{59}$. In addition to RNA plant viruses, certain DNA viruses have been used to deliver

162 genome engineering reagents, including SSNs and DNA templates, for efficient genome editing;

163 readers are directed to the following articles on the development of DNA viruses as vehicles for

164 genome engineering: ${ }^{13,63-66}$.

165

166 4. Using SSNs to develop virus resistance

As noted above, several studies have reported engineering of SSNs to target viral

168 genomes for degradation. Successful attempts used ZFNs to target TYLCV in Arabidopsis ${ }^{67,68}$;

169 resistance developed in transgenic plants expressing ZFNs targeting the TYLCV genome. Initial

170 studies aimed at targeting plant viruses via genome engineering have focused on using ZFN to

171 target begomovirus Rep binding sites/iterons, the iterated nucleotide sequences within the

172 begomovirus genome that provides binding site for virus replication associated protein (Rep).

173 ZFNs developed for iterons of Beet severe curly top virus (BSCTV) and Tomato yellow leaf curl

174 virus (TYLCV) effectively targeted the respective viruses ${ }^{68,69}$. Transgenic Arabidopsis plants

175 expressing ZFNs that target iterons of BSCTV demonstrated complete resistance to this virus ${ }^{69}$.

176 Chen et al. (2014) demonstrated that ZFN can efficiently target BSCTV and TYLCV but argued

177 that this viral sequence-specific strategy may not be effective in the field, where mixed viral

178 infections are common ${ }^{67}$. As an alternative, targeting the conserved regions of viruses would 
179 confer relatively durable resistance. Three conserved regions in Rep of monopartite

180 begomoviruses were identified and tested for broad-spectrum resistance. Among these three

181 targets, one targeting a 25-base pair sequence functioned well against both Tomato yellow leaf

182 curl China virus (TYLCCNV) and Tobacco curly shoot virus (TbCSV) ${ }^{67}$.

Artificial TALE (ATALE), an artificial DNA-binding protein that has a highly conserved

184 central region with tandem repeat units of 34 amino acids, also has been used for designing

185 broad-spectrum resistance to begomoviruses ${ }^{70}$. Two highly conserved targets, AC1 and a

186 nonanucleotide sequence, were selected and targeted using an artificial TALE (ATALE). The

187 DNA binding efficiency of the ATALE was confirmed, and transgenic $N$. benthamiana plants

188 were developed. The authors tested two begomoviruses, Tobacco curly shoot virus (TbCSV) and

189 Tomato leaf curl Yunnan virus (TLCYnV), as well as one begomovirus with its associated

190 betasatellite, Tomato yellow leaf curl China virus (TYLCCNV) with its cognate Tomato yellow

191 leaf curl China betasatellite (TYLCCNB). The resulting transgenic plants demonstrated partial

192 resistance, with delayed symptom development and reduced viral DNA accumulation compared

193 to wild type ${ }^{70}$.

194

195 5. CRISPR/Cas9-mediated virus interference

196 Since the native CRISPR/Cas9 system can confer resistance against invading phages in

197 bacterial and archaeal species, we investigated whether this system could be transferred to plant

198 species to confer molecular immunity against plant DNA viruses just as ZFNs and TALENs

199 were shown to do in the studies discussed above. Among plant DNA viruses, geminiviruses

200 infect several economically important crops such as $\operatorname{cotton}^{71,72}$, tomato ${ }^{73}$, potato ${ }^{74}$, and several 
201 legumes $^{75,76}$, as well as ornamentals ${ }^{77,78}$ and weeds ${ }^{79-81}$. In the past two decades, molecular

202 biologists and breeders have worked to develop geminivirus-resistant crops ${ }^{82,83}$. Recently,

203 several studies have demonstrated that engineering plant species, including tobacco and

204 Arabidopsis, with CRISPR/Cas9 machinery targeting geminiviruses can confer molecular

205 immunity ${ }^{14,16,17}$.

206 We recently showed that engineering plants to express the CRISPR/Cas9 machinery

207 targeting the TYLCV genome led to interference and degradation of the viral genome, resulting

208 in a significant reduction in viral symptoms ${ }^{14}$. In Ali et al., (2015) we showed that $N$.

209 benthamiana plants expressing the CRISPR/Cas9 machinery exhibited resistance against

210 TYLCV, Beet curly top virus (BCTV), and Merremia mosaic virus (MeMV) ${ }^{14}$. Baltes et al. and

211 Ji et al. demonstrated virus interference activities in N. benthamiana against Bean yellow dwarf

212 virus (BeYDV) and Beet severe curly top virus (BSCTV), respectively ${ }^{16,17}$. Ji et al. correlated

213 Cas9 expression with the levels of virus suppression, indicating the need to use a single

214 background transgenic line with optimum expression of Cas9 and sgRNA for practical

215 applications ${ }^{17}$. Baltes et al. showed that one sgRNA targeting the BeYDV genome could confer

216 plant resistance without cleavage activity, suggesting that catalytically inactive Cas9 (dCas9) can

217 be used to mediate virus interference, thereby eliminating concerns about off-target activities in

218 the plant genome ${ }^{16}$.

219 In a follow-up study, we demonstrated that this technology can be used to target and

220 cleave Cotton leaf curl Kokhran virus (CLCuKoV) and that by targeting the conserved

221 nonanucleotide sequence, multiple begomoviruses can be targeted simultaneously (CLCuKoV,

222 TYLCV, TYLCSV, MeMV, BCTV-Worland, and BCTV-Logan), conferring broad-spectrum

223 geminivirus resistance ${ }^{15}$. In these studies, $N$. benthamiana plants expressing the CRISPR/Cas9 
224 system displayed considerably reduced viral titers, which abolished or significantly reduced 225 disease symptoms.

226 We subsequently investigated the differences in efficiency of virus interference when

227 targeting coding versus non-coding sequences ${ }^{15}$. Since invading viruses and host plants exist in

228 an arms race where pathogens rapidly evolve mechanisms to evade plant defenses, we

229 systematically analyzed the ability of geminiviruses to evade the CRISPR/Cas9 machinery. We

230 determined the ability of multiple geminiviruses to evade this machinery by targeting coding and

231 non-coding sequences, finding that targeting coding sequences led to the generation of viral

232 variants capable of evading the CRISPR/Cas9 machinery. Since these viral variants can have

233 variable replication efficiencies, variants with more proficient replication should predominate

234 over those with lower replication rates under natural field conditions, where mixed infections

235 predominate.

236 Interestingly, targeting non-coding intergenic sequences led to high levels of virus

237 interference, with no detectable viral escapes from the CRISPR/Cas9 machinery, thereby

238 providing an effective strategy for conferring potentially durable resistance. Given the

239 considerable heterogeneity and recombination ability of geminiviruses and the ability for

240 replication and systemic movement in CRISPR/Cas9-induced variants, strategies could be

241 devised in which the CRISPR/Cas9 system targets the non-coding region to inhibit or prevent

242 viral replication by mutating the essential parts of geminiviruses. Alternatively, targeting

243 multiple regions of the virus genome simultaneously would abrogate the ability of the virus to

244 use the NHEJ repair system, and these cleaved molecules would ultimately be degraded. 


\section{6. Developing resistance against RNA viruses}

Plant viruses that store and transmit their genetic information in form of RNA are called

248 RNA plant viruses. Several RNA viruses cause devastating yield losses in economically

249 important crops like tomato, potato, cucurbits, etc. Genome engineering strategies aimed at

250 manipulating host factors in the plant genome have been used to develop resistance against RNA

251 viruses ${ }^{84}$. For example, the CRISPR/Cas9 system is used to modify plant genes that affect plant

252 responses to viral infection, thus generating virus resistance. The translation initiation-like

253 factors $e I F 4 E$ and $e I F$ (iso) $4 E$ are directly involved in the infection process of RNA viruses ${ }^{85}$.

254 Arabidopsis plants with mutations in these translation initiation factors exhibit resistance to

255 Turnip mosaic virus (TuMV) ${ }^{86}$. Therefore, these targets were mutated using the CRISPR/Cas9

256 system to develop virus-resistant plants ${ }^{84,87}$. The utility of CRISPR/Cas9 technology for

257 generating novel genetic resistance to the potyvirus TuMV was demonstrated in Arabidopsis via

258 deletion of the host factor, $e I F(i s o) 4 E$, which is strictly required for viral survival ${ }^{84}$. Virus

259 resistance was also developed in cucumber (Cucumis sativus) by disrupting eIF4E, resulting in

260 non-transgenic, heterozygous eIF4E mutant plants. These non-transgenic plants exhibited partial

261 resistance to an ipomovirus (Cucumber vein yellowing virus) and two potyviruses (Zucchini

262 yellow mosaic virus and Papaya ring spot mosaic virus-W) ${ }^{87}$.

263 Translation initiation factor genes are prime candidates for host genes that can be targeted

$264{ }^{85}$, but any host gene encoding a factor that the virus requires is a potential target for

265 modification. This form of recessive resistance could be exploited with the aid of the

266 CRISPR/Cas9 system to create novel resistance alleles in crop plants to protect them against

267 problematic viruses that use host translation initiation factors. 


\section{7. Outlook}

The CRISPR/Cas9 system has recently emerged as a groundbreaking genome-

271 engineering platform. This system has been used to improve several traits in plants, e.g.,

272 engineering biotic stress tolerance (against invading viruses ${ }^{14-16,81,83,88}$, fungus ${ }^{89,90}$, bacteria, and

273 insects), abiotic stress tolerance ${ }^{91}$ (against drought ${ }^{54}$, heat, and cold stress), and yield

274 improvement ${ }^{34}$ (e.g., by increasing grain weight in maize ${ }^{54}$ ). Moreover, all of these works can be

275 performed using a "DNA-free", non-genetically modified organisms (GMO) crop production

276 system ${ }^{92,93}$. In addition to crop improvement, the CRISPR/Cas9 system can be used to examine

277 the plant genome, e.g., by creating knockout libraries or by enhancing plant genome imaging ${ }^{94}$

278 (Figure 4). The CRISPR/Cas9 system has been used for targeted genome editing in diverse plant

279 species, from model plants such as N. benthamiana and Arabidopsis to economically important

280 plants such as wheat, maize, sorghum, and rice ${ }^{94}$. The specificity of this system is determined by

281 the 20-nucleotide sequence of the sgRNA, allowing for unprecedented, simple genome

282 engineering. Furthermore, the CRISPR/Cas9 system can be used to simultaneously edit multiple

283 genomic targets ${ }^{95}$.

Viruses have long been used for genome engineering, mainly as delivery vehicles for mammalian cells ${ }^{6}$. Plant viruses can potentially be powerful tools for improving the efficiency

286 of genome editing ${ }^{12,13}$. Recent studies have demonstrated the utility and applicability of using 287 viruses for genome editing via CRISPR/Cas9 genome engineering platforms ${ }^{94}$. In these studies, 288 to generate plants with heritable modifications, it was necessary to generate transgenic lines that 289 stably expressed Cas9 and gRNA; progeny with targeted modifications were then recovered in 290 subsequent generations. Since producing transgenic plants is time-consuming, efficient delivery 
291 methods are needed to expedite and maximize the utility of this technology for trait discovery

292 and development. The recently developed TRV-mediated CRISPR/Cas9 delivery system ${ }^{12}$ can

293 help overcome this hurdle. The use of heterozygous Cas9-overexpressing plants with this simple,

294 versatile genome-editing platform allows plants free of foreign DNA to be generated, which

295 might help overcome the regulatory hurdles that impede the commercialization of genetically

296 engineered plants. TRV has multiple advantages as a delivery vehicle for sgRNA, including its

297 small RNA genome, which is amenable to library construction and does not integrate into the

298 host genome. In addition, TRV can infect germline cells and has a wide host range under both

299 natural and laboratory conditions ${ }^{96}$. However, the limitations in the carrying capacity of TRV

300 and other RNA viruses prevent their use beyond the expression of relatively small SSNs and

301 sgRNAs, and they are unable to efficiently deliver DNA repair templates. Perhaps geminiviruses

302 can be used to overcome the limitations of RNA viruses due to their larger carrying capacity and

303 their ability to produce a DNA replicon capable of acting as a repair template for gene targeting

$304{ }^{97}$. In an effort to facilitate efficient genome engineering, various viruses have recently been

305 developed to deliver genome-engineering reagents into plants ${ }^{12,13,63-66}$.

Several genome-engineering platforms have been used to engineer plants with molecular

307 immunity against invading viruses. However, the CRISPR/Cas9 system has shown promise for

308 providing immunity against economically important, widespread geminiviruses such as TYLCV.

309 Moreover, targeting the non-coding regions of viruses provides significant interference activity

310 and limits the generation of viral variants that escape the CRISPR/Cas9 machinery and are

311 capable of replication and systemic movement ${ }^{15}$. These developments are exciting but have thus

312 far been limited to laboratory demonstrations. Field trials in multiple locations would be helpful

313 for further evaluating the actual success of this technology under natural conditions. Moreover, 
314 previous studies have mainly focused on targeting DNA viruses due to the robust activity of the

315 CRISPR/Cas9 system for cleaving DNA substrates. Novel variants of the CRISPR/Cas system

316 were recently shown to be capable of targeting RNA genomes ${ }^{98}$. These systems are besed on the

317 concept that the Cas9 binds with high affinity to single-stranded RNA (ssRNA) targets matching

318 the Cas9-associated gRNA sequence when the PAM is presented in trans as a separate DNA

319 oligonucleotide. Furthermore, PAM-presenting oligonucleotides (PAMmers) stimulate site-

320 specific endonucleolytic cleavage of ssRNA targets; using specially designed PAMmers, Cas9

321 can be specifically directed to bind or cut RNA targets ${ }^{98}$. Such systems could be applied to target

322 the RNA genomes of single and multiple viruses and to confer resistance against RNA viruses.

One of the salient features of the CRISPR/Cas9 system is that it can be used in a 'DNA-

324 Free' crop production system to develop non-transgenic plants with desired traits ${ }^{99}$. This is even

325 more practical when endogenous host genes are targeted, since the progeny plants in the

326 following segregating generations can be selected for the desired phenotypes caused by the

327 edited traits, but lacking the Cas 9 and sgRNA transgenes ${ }^{100}$. This system has direct application in

328 crop production and variety development ${ }^{89,93}$. An alternate approach to avoid the incorporation

329 of foreign genes into the host genome can be the introduction of CRISPR/Cas9 reagents directly

330 into the cell, for example by using a Cas $9 / \mathrm{sgRNA}$ ribonucleoprotein complex ${ }^{99,101-103}$. In this way

331 the final product would be a plant, no different from the one produced as a result of classical

332 plant breeding approaches ${ }^{104}$. The DNA-free mushroom and maize varieties, that have been

333 developed using similar CRISPR/Cas9 mediated genome editing approach have been deemed by

334 the US Department of Agriculture to not be subject to their regulatory system ${ }^{92,93}$. This decision

335 may pave the way for these and other plants free of foreign DNAs to enter the marketplace.

336 Overall these strategies can be used to devise a variety development system that might bypass 
337 GMO regulations (Figure 4) ${ }^{105}$. However, the actual regulatory status of most genome-edited 338 crops is still under debate ${ }^{105}$.

\section{References}

341 1. Stella S, Montoya G. The genome editing revolution: A CRISPR-Cas TALE off-target $342 \quad$ story. Bioessays. Jul 2016;38(S1):S4-S13.

343 2. Weeks DP, Spalding MH, Yang B. Use of designer nucleases for targeted gene and 344 genome editing in plants. Plant Biotechnology Journal. 2016;14(2):483-495.

345 3. Price AA, Grakoui A, Weiss DS. Harnessing the Prokaryotic Adaptive Immune System 346 as a Eukaryotic Antiviral Defense. Trends in Microbiology. 4// 2016;24(4):294-306.

347 4. Mahfouz MM, Piatek A, Stewart CN, Jr. Genome engineering via TALENs and 348 CRISPR/Cas9 systems: challenges and perspectives. Plant Biotechnol J. Oct $349 \quad 2014 ; 12(8): 1006-1014$.

350 5. Weeks DP, Jiang W. Genome Editing in Plants Using CRISPR/Cas9/sgRNA 351 Technologies. Paper presented at: IN VITRO CELLULAR \& DEVELOPMENTAL $352 \quad$ BIOLOGY-ANIMAL, 2014.

353 6. Chen X, Goncalves MAFV. Engineered Viruses as Genome Editing Devices. Mol Ther. $354 \quad$ 03//print 2016;24(3):447-457.

355 7. Ellis J, Bernstein A. Gene targeting with retroviral vectors: recombination by gene 356 conversion into regions of nonhomology. Mol Cell Biol. Apr 1989;9(4):1621-1627. 
357 8. Lombardo A, Genovese P, Beausejour CM, et al. Gene editing in human stem cells using zinc finger nucleases and integrase-defective lentiviral vector delivery. Nature

12. Ali Z, Abul-faraj A, Li L, et al. Efficient virus-mediated genome editing in plants using the CRISPR/Cas9 system. Mol Plant. Aug 2015;8(8):1288-1291.

13. Cermak T, Baltes NJ, Cegan R, Zhang Y, Voytas DF. High-frequency, precise modification of the tomato genome. Genome Biol. Nov 06 2015;16:232.

14. Ali Z, Abulfaraj A, Idris A, Ali S, Tashkandi M, Mahfouz MM. CRISPR/Cas9-mediated viral interference in plants. Genome Biol. Nov 11 2015;16(1):238.

15. Ali Z, Ali S, Tashkandi M, Zaidi SS, Mahfouz MM. CRISPR/Cas9-mediated immunity to geminiviruses: Differential interference and evasion. Sci Rep. May 26 2016;6:26912.

16. Baltes NJ, Hummel AW, Konecna E, et al. Conferring resistance to geminiviruses with the CRISPR-Cas prokaryotic immune system. Nat Plants. Sep 28 2015;1(10):15145.

17. Ji X, Zhang H, Zhang Y, Wang Y, Gao C. Establishing a CRISPR-Cas-like immune system conferring DNA virus resistance in plants. Nat Plants. Sep 28 2015;1(10):15144.

18. Li T, Liu B, Spalding MH, Weeks DP, Yang B. High-efficiency TALEN-based gene editing produces disease-resistant rice. Nat Biotech. 05//print 2012;30(5):390-392. 
380

381

382

383

384

385

386

387

388

389

390

391

392

393

394

395

396

397

398

399

400

401

19. Aouida M, Li L, Mahjoub A, et al. Transcription activator-like effector nucleases mediated metabolic engineering for enhanced fatty acids production in Saccharomyces cerevisiae. Journal of Bioscience and Bioengineering. 10// 2015;120(4):364-371.

20. Li T, Huang S, Jiang WZ, et al. TAL nucleases (TALNs): hybrid proteins composed of TAL effectors and FokI DNA-cleavage domain. Nucleic Acids Res. Jan 2011;39(1):359372.

21. Li T, Huang S, Zhao X, et al. Modularly assembled designer TAL effector nucleases for targeted gene knockout and gene replacement in eukaryotes. Nucleic acids research. 2011:gkr188.

22. Li T, Liu B, Chen CY, Yang B. TALEN-Mediated Homologous Recombination Produces Site-Directed DNA Base Change And Herbicide-Resistant Rice. J Genet Genomics. Mar 222016.

23. Li T, Liu B, Chen CY, Yang B. TALEN utilization in rice genome modifications. Methods. Aug 15 2014;69(1):9-16.

24. Brazelton VA, Zarecor S, Wright DA, et al. A quick guide to CRISPR sgRNA design tools. GM Crops \& Food. 2015/10/02 2015;6(4):266-276.

25. Eid A, Mahfouz MM. Genome editing: the road of CRISPR/Cas9 from bench to clinic. Exp Mol Med. 10/14/online 2016;48:e265.

26. Wright AV, Nunez JK, Doudna JA. Biology and applications of CRISPR systems: Harnessing nature's toolbox for genome engineering. Cell. Jan 14 2016;164(1-2):29-44.

27. Voytas DF. Plant genome engineering with sequence-specific nucleases. Annu Rev Plant Biol. 2013;64(1):327-350. 
402

403

404

405

406

407

408

409

410

411

412

413

414

415

416

417

418

419

420

421

422

423

28. Shukla VK, Doyon Y, Miller JC, et al. Precise genome modification in the crop species Zea mays using zinc-finger nucleases. Nature. May 21 2009;459(7245):437-441.

29. Townsend JA, Wright DA, Winfrey RJ, et al. High-frequency modification of plant genes using engineered zinc-finger nucleases. Nature. May 21 2009;459(7245):442-445.

30. Li L, Atef A, Piatek A, et al. Characterization and DNA-binding specificities of Ralstonia TAL-like effectors. Mol Plant. Jul 2013;6(4):1318-1330.

31. Ji Z, Ji C, Liu B, Zou L, Chen G, Yang B. Interfering TAL effectors of Xanthomonas oryzae neutralize R-gene-mediated plant disease resistance. Nature Communications. 11/04/online 2016;7:13435.

32. Piatek A, Mahfouz MM. Targeted genome regulation via synthetic programmable transcriptional regulators. Crit Rev Biotechnol. Apr 19 2016:1-12.

33. Aouida M, Piatek MJ, Bangarusamy DK, Mahfouz MM. Activities and specificities of homodimeric TALENs in Saccharomyces cerevisiae. Curr Genet. May 2014;60(2):61-74.

34. Jiang WZ, Henry IM, Lynagh PG, Comai L, Cahoon EB, Weeks DP. Significant enhancement of fatty acid composition in seeds of the allohexaploid, Camelina sativa, using CRISPR/Cas9 gene editing. Plant Biotechnology Journal. 2016:n/a-n/a.

35. Jiang W, Brueggeman AJ, Horken KM, Plucinak TM, Weeks DP. Successful transient expression of Cas9 and single guide RNA genes in Chlamydomonas reinhardtii. Eukaryotic cell. 2014;13(11):1465-1469.

36. Harms DW, Quadros RM, Seruggia D, et al. Mouse Genome Editing Using the CRISPR/Cas System. Current Protocols in Human Genetics: John Wiley \& Sons, Inc.; 2001. 
424 37. Housden BE, Perrimon N. Comparing CRISPR and RNAi-based screening technologies.

425

426

427

428

429

430

431

432

433

434

435

436

437

438

439

440

441

442

443

444

445

446

Nat Biotech. 06//print 2016;34(6):621-623.

38. Barrangou R, Doudna JA. Applications of CRISPR technologies in research and beyond. Nat Biotech. 09//print 2016;34(9):933-941.

39. Horvath P, Barrangou R. CRISPR/Cas, the immune system of bacteria and archaea. Science. 2010;327(5962):167-170.

40. Barrangou R, Fremaux C, Deveau H, et al. CRISPR provides acquired resistance against viruses in prokaryotes. Science. 2007;315(5819):1709-1712.

41. Gasiunas G, Barrangou R, Horvath P, Siksnys V. Cas9-crRNA ribonucleoprotein complex mediates specific DNA cleavage for adaptive immunity in bacteria. Proceedings of the National Academy of Sciences. 2012;109(39):E2579-E2586.

42. Horvath P, Romero DA, Coûté-Monvoisin A-C, et al. Diversity, activity, and evolution of CRISPR loci in Streptococcus thermophilus. Journal of bacteriology. 2008;190(4):14011412.

43. Mojica F, Diez-Villasenor C, Garcia-Martinez J, Almendros C. Short motif sequences determine the targets of the prokaryotic CRISPR defence system. Microbiology. 2009;155(3):733-740.

44. Datsenko KA, Pougach K, Tikhonov A, Wanner BL, Severinov K, Semenova E. Molecular memory of prior infections activates the CRISPR/Cas adaptive bacterial immunity system. Nature Communications. 07/10/online 2012;3:945.

45. Sapranauskas R, Gasiunas G, Fremaux C, Barrangou R, Horvath P, Siksnys V. The Streptococcus thermophilus CRISPR/Cas system provides immunity in Escherichia coli. Nucleic acids research. 2011:gkr606. 
447

448

449

450

451

452

453

454

455

456

457

458

459

460

461

462

463

464

465

466

467

468

469

46. Jiang W, Zhou H, Bi H, Fromm M, Yang B, Weeks DP. Demonstration of CRISPR/Cas9/sgRNA-mediated targeted gene modification in Arabidopsis, tobacco, sorghum and rice. Nucleic acids research. 2013:gkt780.

47. Ran FA, Hsu PD, Wright J, Agarwala V, Scott DA, Zhang F. Genome engineering using the CRISPR-Cas9 system. Nat. Protocols. 11//print 2013;8(11):2281-2308.

48. Zhou H, Liu B, Weeks DP, Spalding MH, Yang B. Large chromosomal deletions and heritable small genetic changes induced by CRISPR/Cas9 in rice. Nucleic Acids Res. 2014;42(17):10903-10914.

49. Paul JW, 3rd, Qi Y. CRISPR/Cas9 for plant genome editing: accomplishments, problems and prospects. Plant Cell Rep. Apr 25 2016:1-11.

50. Eid A, Ali Z, Mahfouz MM. High efficiency of targeted mutagenesis in arabidopsis via meiotic promoter-driven expression of Cas9 endonuclease. Plant Cell Rep. May 282016.

51. Jiang W, Yang B, Weeks DP. Efficient CRISPR/Cas9-Mediated Gene Editing in Arabidopsis thaliana and Inheritance of Modified Genes in the T2 and T3 Generations. PLOS ONE. 2014;9(6):e99225.

52. Zhang $\mathrm{Y}$, Liang Z, Zong $\mathrm{Y}$, et al. Efficient and transgene-free genome editing in wheat through transient expression of CRISPR/Cas9 DNA or RNA. Nat Commun. 08/25/online $2016 ; 7: 12617$.

53. Char SN, Neelakandan AK, Nahampun H, et al. An Agrobacterium-delivered CRISPR/Cas9 system for high-frequency targeted mutagenesis in maize. Plant Biotechnol J. Aug 112016.

54. Shi J, Gao H, Wang H, et al. ARGOS8 variants generated by CRISPR-Cas9 improve maize grain yield under field drought stress conditions. Plant Biotechnol J. Jul 212016. 
470 55. Mahfouz MM, Cardi T, Neal Stewart C. Next-generation precision genome engineering 471 and plant biotechnology. Plant Cell Reports. 2016;35(7):1397-1399.

472 56. Piatek $A$, Ali $Z$, Baazim $H$, et al. RNA-guided transcriptional regulation in planta via synthetic dCas9-based transcription factors. Plant Biotechnol J. May 2015;13(4):578-

57. Aouida M, Eid A, Ali Z, et al. Efficient fdCas9 Synthetic Endonuclease with Improved Specificity for Precise Genome Engineering. PLOS ONE. 2015;10(7):e0133373.

477 58. Xu S, Cao S, Zou B, et al. An alternative novel tool for DNA editing without target sequence limitation: the structure-guided nuclease. Genome Biology. 2016;17(1):186.

59. Marton I, Zuker A, Shklarman E, et al. Nontransgenic genome modification in plant cells. Plant Physiol. Nov 2010;154(3):1079-1087.

60. Ali Z, Abul-Faraj A, Piatek M, Mahfouz MM. Activity and specificity of TRV-mediated gene editing in plants. Plant Signaling and Behavior. 2015/10/03 2015;10(10):e1044191.

61. Shivprasad S, Pogue GP, Lewandowski DJ, et al. Heterologous Sequences Greatly Affect Foreign Gene Expression in Tobacco Mosaic Virus-Based Vectors. Virology. 1999/03/15 $1999 ; 255(2): 312-323$.

62. Choi IR, Stenger DC, Morris TJ, French R. A plant virus vector for systemic expression of foreign genes in cereals. Plant J. Aug 2000;23(4):547-555.

63. Gil-Humanes J, Wang Y, Liang Z, et al. High efficiency gene targeting in hexaploid wheat using DNA replicons and CRISPR/Cas9. Plant J. Dec 10 2016;DOI: 10.1111/tpj.13446. 
491

492

493

494

495

496

497

498

499

500

501

502

503

504

505

506

507

508

509

510

511

512

64. Butler NM, Baltes NJ, Voytas DF, Douches DS. Geminivirus-mediated genome editing in potato (Solanum tuberosum L.) using sequence-specific nucleases. Front Plant Sci. 2016-July-21 2016;7(1045):1045.

65. Butler NM, Atkins PA, Voytas DF, Douches DS. Generation and inheritance of targeted mutations in potato (Solanum tuberosum L.) using the CRISPR/Cas system. Plos One. Dec 14 2015;10(12):e0144591.

66. Baltes NJ, Gil-Humanes J, Cermak T, Atkins PA, Voytas DF. DNA replicons for plant genome engineering. Plant Cell. Jan 2014;26(1):151-163.

67. Chen W, Qian Y, Wu X, Sun Y, Wu X, Cheng X. Inhibiting replication of begomoviruses using artificial zinc finger nucleases that target viral-conserved nucleotide motif. Virus Genes. 2014;48(3):494-501.

68. Mori T, Takenaka K, Domoto F, Aoyama Y, Sera T. Inhibition of binding of tomato yellow leaf curl virus rep to its replication origin by artificial zinc-finger protein. $\mathrm{Mol}$ Biotechnol. Jun 2013;54(2):198-203.

69. Sera T. Inhibition of virus DNA replication by artificial zinc finger proteins. $J$ Virol. 2005;79:2614-2619.

70. Cheng X, Li F, Cai J, et al. Artificial TALE as a convenient protein platform for engineering broad-spectrum resistance to begomoviruses. Viruses. Aug 2015;7(8):47724782.

71. Zaidi SSA, Iqbal Z, Amin I, Mansoor S. First report of Tomato leaf curl Gujarat virus, a bipartite begomovirus on cotton showing leaf curl symptoms in Pakistan. Plant Dis. Nov 2015;99(11):1655-1656. 
513 72. Zaidi SS, Shafiq M, Amin I, et al. Frequent occurrence of Tomato leaf curl New Delhi

514 virus in cotton leaf curl disease affected cotton in Pakistan. PLoS One. 2016;11(5):e0155520.

516 73. Zaidi SS, Martin DP, Amin I, Farooq M, Mansoor S. Tomato leaf curl New Delhi virus: a widespread bipartite begomovirus in the territory of monopartite begomoviruses. Mol Plant Pathol. Aug 23 2016;doi: 10.1111/mpp.12481.

74. Hameed A, Tahir MN, Amin I, Mansoor S. First report of Tomato leaf curl New Delhi virus and a Tomato yellow leaf curl Thailand betasatellite causing severe leaf curl disease

522 75. Zaidi SS, Amin I, Iqbal Z, Akhtar KP, Scheffler BE, Mansoor S. Sesbania bispinosa, a 523 new host of a begomovirus-betasatellite complex in Pakistan. Can J Plant Pathol.

525 76. Zaidi SS-e-A, Shakir S, Farooq M, Amin I, Mansoor S. First report of a novel strain of 526 Tomato yellow leaf curl virus-Pakistan causing yellow leaf curl disease on cluster bean in Pakistan. Plant Disease. 2017.

77. Khatri S, Nahid N, Fauquet CM, Mubin M, Nawaz-ul-Rehman MS. A betasatellitedependent begomovirus infects ornamental rose: characterization of begomovirus infecting rose in Pakistan. Virus Genes. Aug 2014;49(1):124-131.

531 78. Mahesh YS, Shankarappa KS, Rangaswamy KT, Prameela HA, Aswathanarayana DS, 532 Maruthi MN. Detection of a begomovirus associated with leaf curl disease of ornamental 533 croton (Codieum variegatum) in southern India. Journal of Plant Pathology. 2008;90:143. 
535 79. Zaidi SS, Shakir S, Farooq M, Amin I, Mansoor S. First Report of Alternanthera yellow 536 vein virus From Eclipta prostrata in Pakistan. Plant Disease. 2017;101(1):266-266.

537 80. Zaidi SS-e-A, Shakir S, Malik HJ, Farooq M, Amin I, Mansoor S. First report of Tomato 538 leaf curl New Delhi virus on Calotropis procera in Pakistan. Plant Disease. 2017.

539 81. Zaidi SS, Mansoor S, Ali Z, Tashkandi M, Mahfouz MM. Engineering Plants for 540 Geminivirus Resistance with CRISPR/Cas9 System. Trends Plant Sci. Apr $541 \quad 2016 ; 21(4): 279-281$.

542 82. Zaidi SS, Briddon RW, Mansoor S. Engineering dual Begomovirus-Bemisia tabaci $543 \quad$ resistance in plants. Trends Plant Sci. 2017;22(1):6-8.

544 83. Zaidi SS, Tashkandi M, Mansoor S, Mahfouz MM. Engineering plant immunity: Using 545 CRISPR/Cas9 to generate virus resistance. Front Plant Sci. 08-Nov-2016 2016;7:1673.

84. Pyott DE, Sheehan E, Molnar A. Engineering of CRISPR/Cas9-mediated potyvirus resistance in transgene-free Arabidopsis plants. Mol Plant Pathol. Oct 2016;17(8):12761288.

85. Sanfacon H. Plant translation factors and virus resistance. Viruses. Jul 2015;7(7):33923419.

551 86. Lellis AD, Kasschau KD, Whitham SA, Carrington JC. Loss-of-susceptibility mutants of 552 Arabidopsis thaliana reveal an essential role for eIF(iso)4E during potyvirus infection.

554 87. Chandrasekaran J, Brumin M, Wolf D, et al. Development of broad virus resistance in 555 non-transgenic cucumber using CRISPR/Cas9 technology. Mol Plant Pathol. Sep 556 2016;17(7):1140-1153. 
557 88. Subburaj S, Tu L, Jin Y-T, Bae S, Lee G-J. Development of Potyvirus Resistance in Non558 transgenic Tomato Plants Using CRISPR/Cas9 Technology. Paper presented at: 2016 한국육종학회-차세대 BG21 사업단-GSP 사업단 공동심포지엄, 2016.

560

561

562

563

564

565

566

567

568

569

570

571

572

573

574

575

576

577

89. Wang Y, Cheng X, Shan Q, et al. Simultaneous editing of three homoeoalleles in hexaploid bread wheat confers heritable resistance to powdery mildew. Nat Biotech. 09//print 2014;32(9):947-951.

90. Wang F, Wang C, Liu P, et al. Enhanced Rice Blast Resistance by CRISPR/Cas9Targeted Mutagenesis of the ERF Transcription Factor Gene OsERF922. PLoS One. 2016;11(4):e0154027.

91. Osakabe Y, Watanabe T, Sugano SS, et al. Optimization of CRISPR/Cas9 genome editing to modify abiotic stress responses in plants. Sci Rep. 2016;6:26685.

92. Waltz E. CRISPR-edited crops free to enter market, skip regulation. Nat Biotech. 06//print 2016;34(6):582-582.

93. Waltz E. Gene-edited CRISPR mushroom escapes US regulation. Nature. Apr 21 2016;532(7599):293.

94. Noman A, Aqeel M, He S. CRISPR-Cas9: Tool for qualitative and quantitative plant genome editing. Front Plant Sci. 2016;7(1740):1740.

95. Cong L, Ran FA, Cox D, et al. Multiplex genome engineering using CRISPR/Cas systems. Science. Feb 15 2013;339(6121):819-823.

96. Senthil-Kumar M, Mysore KS. Tobacco rattle virus-based virus-induced gene silencing in Nicotiana benthamiana. Nat Protoc. Jul 2014;9(7):1549-1562. 
97. Richter KS, Kleinow T, Jeske H. Somatic homologous recombination in plants is promoted by a geminivirus in a tissue-selective manner. Virology. 2014;452-453:287296.

98. O'Connell MR, Oakes BL, Sternberg SH, East-Seletsky A, Kaplan M, Doudna JA. Programmable RNA recognition and cleavage by CRISPR/Cas9. Nature. Dec 11 2014;516(7530):263-266.

99. Woo JW, Kim J, Kwon SI, et al. DNA-free genome editing in plants with preassembled CRISPR-Cas9 ribonucleoproteins. Nat Biotechnol. 11//print 2015;33(11):1162-1164.

100. Kanchiswamy CN. DNA-free genome editing methods for targeted crop improvement. Plant Cell Rep. Apr 21 2016;35(7):1469-1474.

101. Kim J, Kim J-S. Bypassing GMO regulations with CRISPR gene editing. Nat Biotech. 10//print 2016;34(10):1014-1015

102. Malnoy M, Viola R, Jung M-H, et al. DNA-Free Genetically Edited Grapevine and Apple Protoplast Using CRISPR/Cas9 Ribonucleoproteins. Frontiers in Plant Science. 2016December-20 2016;7(1904).

103. Liang Z, Chen K, Li T, et al. Efficient DNA-free genome editing of bread wheat using CRISPR/Cas9 ribonucleoprotein complexes. Nature Communications. 01/18/online 2017;8:14261.

104. Voytas DF, Gao C. Precision genome engineering and agriculture: Opportunities and regulatory challenges. PLoS Biol. Jun 2014;12(6):e1001877.

105. Wolt JD, Wang K, Yang B. The Regulatory Status of Genome-edited Crops. Plant Biotechnology Journal. 2016;14(2):510-518. 
602 Figure legends

603 Figure 1. Genome engineering platforms and their utilization.

604 The zinc finger nuclease (ZFN) system is composed of a zinc finger domain and a FokI

605 endonuclease domain. The trinucleotide binding sites within each zinc finger domain dictate the

606 specificity of DNA binding, while coupling of the nuclease domains (derived from the type II

607 restriction endonuclease FokI) of two ZFNs produces a double stranded break (DSB) at the

608 designated target site. Transcription activator-like effector nucleases (TALENs) also contains

609 two domains, a DNA binding domain and a FokI endonuclease domain. But in this case the DNA

610 binding specificity is dictated by the TALE array that contains 33-34 nucleotide conserved

611 residue repetitive motifs. These motifs contain repeated variable di-residues (RVDs) at positions

612 twelve and thirteen that specify binding to either an A, G, C or T nucleotide in the DNA target

613 site. Similar to ZNF, the dimerized FokI endonuclease produces a DSB at the target site. The

614 clustered regularly interspaced short palindromic repeat (CRISPR)/CRISPR associated 9 (Cas9)

615 system consists of Cas9 endonuclease and a single guide RNA ( $\operatorname{sgRNA}$ ). In case of

616 CRISPR/Cas9, the DNA binding specificity is dictated by specific hybridization to the sgRNA

617 where the two Cas9 nuclease domains produce a DSB three base pairs upstream of the

618 protospacer adjacent motif (PAM). The repair of the DSB is either by the error-prone non-

619 homologous end joining (NHEJ) system or by homology-directed repair (HDR). During NHEJ,

620 random insertions or deletions can occur, which ultimately may lead to gene disruption. By

621 contrast, HDR uses a donor DNA template that can be used to direct gene replacement/addition

622 or gene sequence modification. 
624 Figure 2. Molecular basis of CRISPR/Cas9-mediated genome engineering using the TRV

625 system.

626 The engineered TRV genome is transcribed inside the plant cell's nucleus, producing a single

627 guide RNA (sgRNA) under the control of a user-defined promoter. The target plant must be

628 genetically engineered with a Cas9 gene to constitutively produce the Cas9 endonuclease. Cas9

629 binds the TRV-encoded sgRNA and cleaves the plant genome at the gRNA-directed site (red

630 arrow), three base pairs upstream of the PAM sequence. The resulting double-stranded break

631 (DSB) is repaired by the cell's error-prone non-homologous end joining (NHEJ) DNA repair

632 system. As a result, certain insertions or deletions (indels) are produced, ultimately disrupting the

633 target gene (in most cases). This engineered mutation is a permanent part of the plant's genome

634 and any resulting altered phenotype is transmissible to the next generation.

635

636 Figure 3. Stepwise methodology for TRV-mediated genome engineering.

637 In the TRV2 genome, a 20-nucleotide target sequence preceding the PAM sequence is cloned 638 into the sgRNA gene that is under the control of an appropriate promoter. Agrobacterium

639 cultures carrying DNA segments encoding the engineered TRV2 genome (conferring user-

640 selected sequence specificity) and the TRV1 genome are co-infiltrated into the leaves of plants

641 overexpressing Cas9 (Cas9-OE) via agroinfection. For example in figure the sgRNA (cloned in

642 TRV2) is designed to target a malfunctioning gene makes plant leaves yellow. After

643 agroinfection, the plants are analyzed for the presence of the targeted modification that restors

644 the green leaf phenotype. Plant leaf discs carrying modified genomes can be regenerated to 
645 recover mutant plants, or the seed progeny can be screened for the presence of the modification

646 in the next generation., thereby bypassing the need for tissue culture.

647

648 Figure 4. Applications of CRISPR/Cas9 in plants

649 CRISPR/Cas9 can be (and has been) used to improve several plant traits, e.g., engineering biotic

650 stress tolerance (resistance against invading viruses, bacteria, and insects), abiotic stress

651 tolerance (resistance against drought, heat, and cold stress) and yield improvement (e.g., by

652 increasing grain weight in wheat, kernel size in corn, and the number of bolls in cotton).

653 Moreover, all of this work can be performed in a "DNA-free", non-GMO crop production

654 system. In addition to crop improvement, the CRISPR/Cas9 system can be used to greatly

655 accelerate fundamental plant research, e.g., by constructing extensive gene knockout libraries or

656 by producing plants with key gene replacements or modifications.

657 Figure 1:

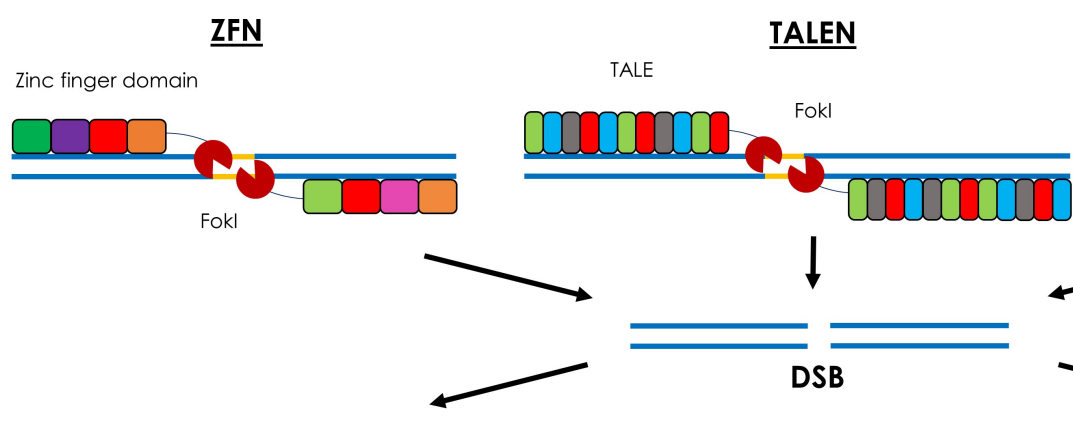

NHEJ

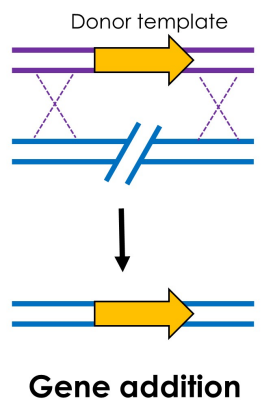

$\underline{\text { HDR }}$
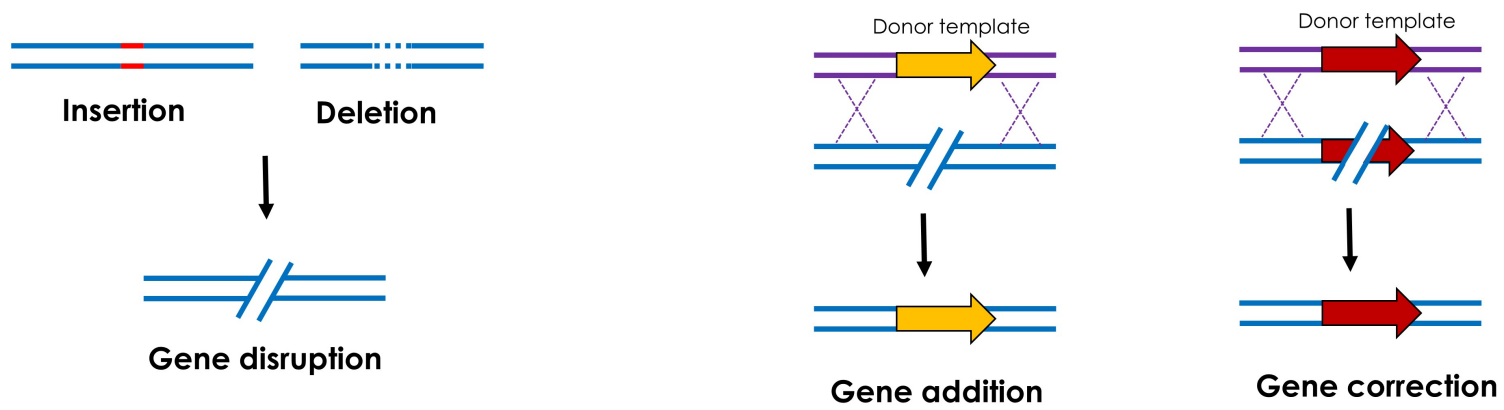


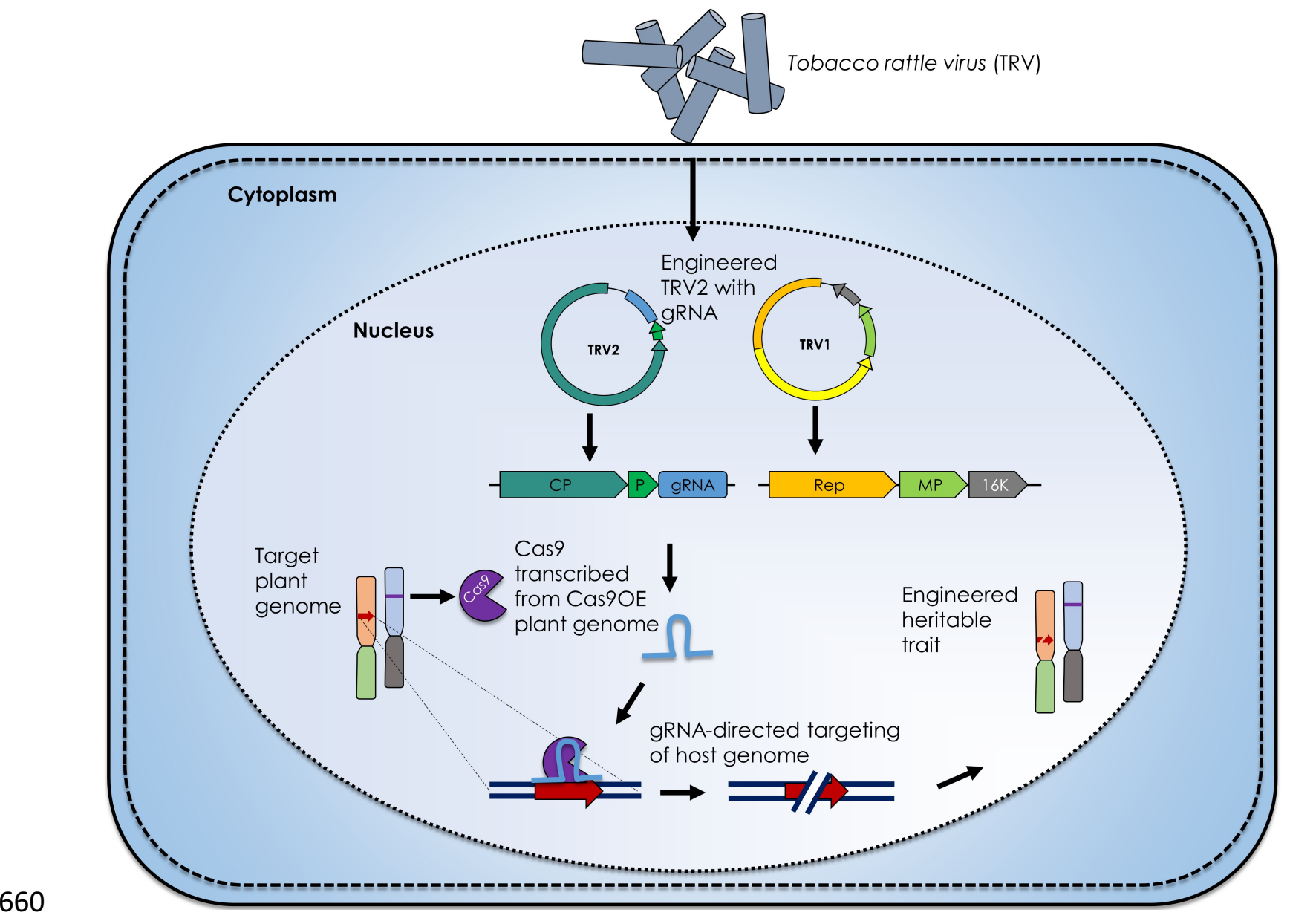


661 Figure 3:

Page 32 of $\mathbf{3 4}$ 
Cas9 overexpression plant with target genes

$T R V$, with gRNA for target gene/s, is agro infiltrated; gene editing starts (green leaf)
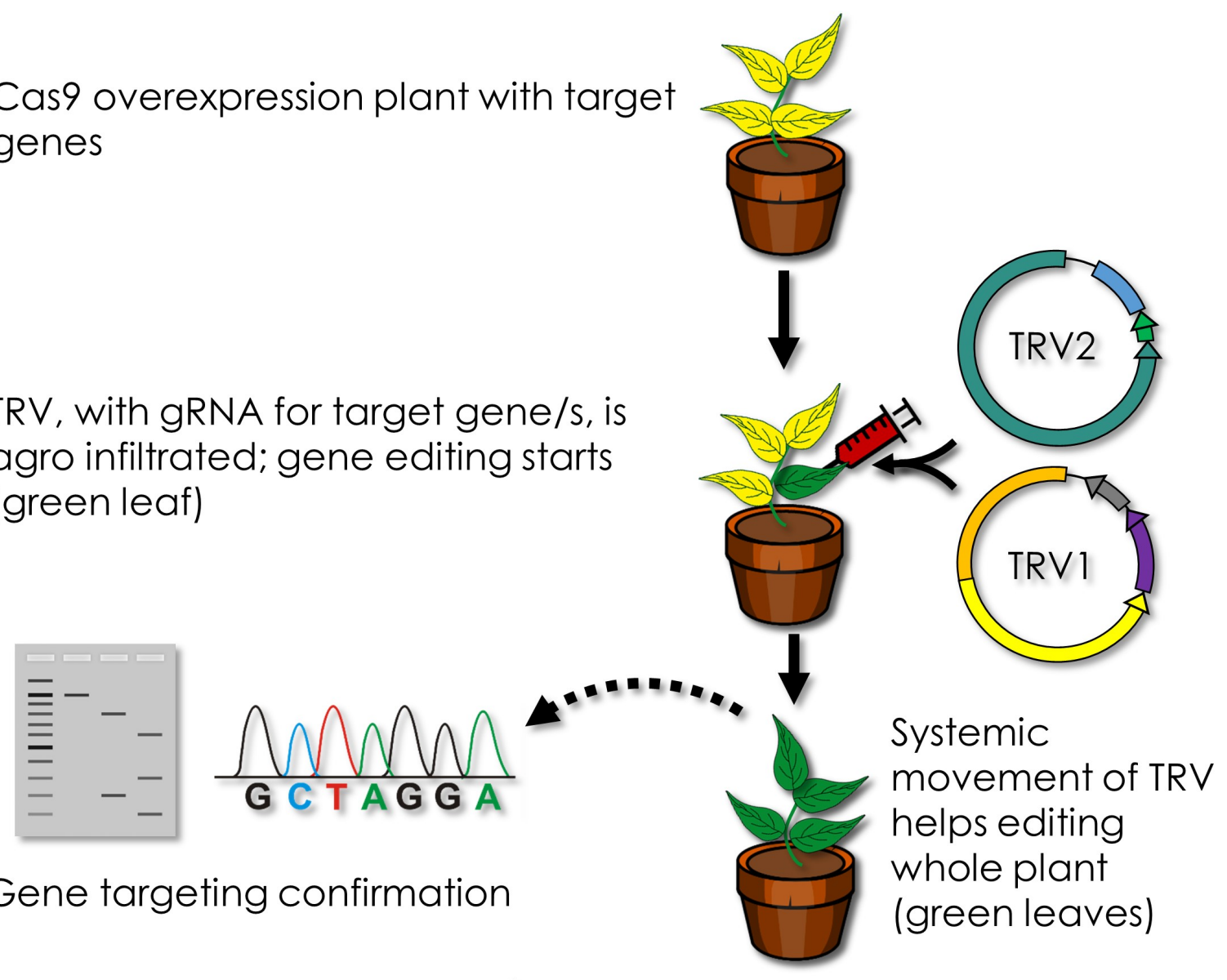

Tissue cultureindependent genome engineering

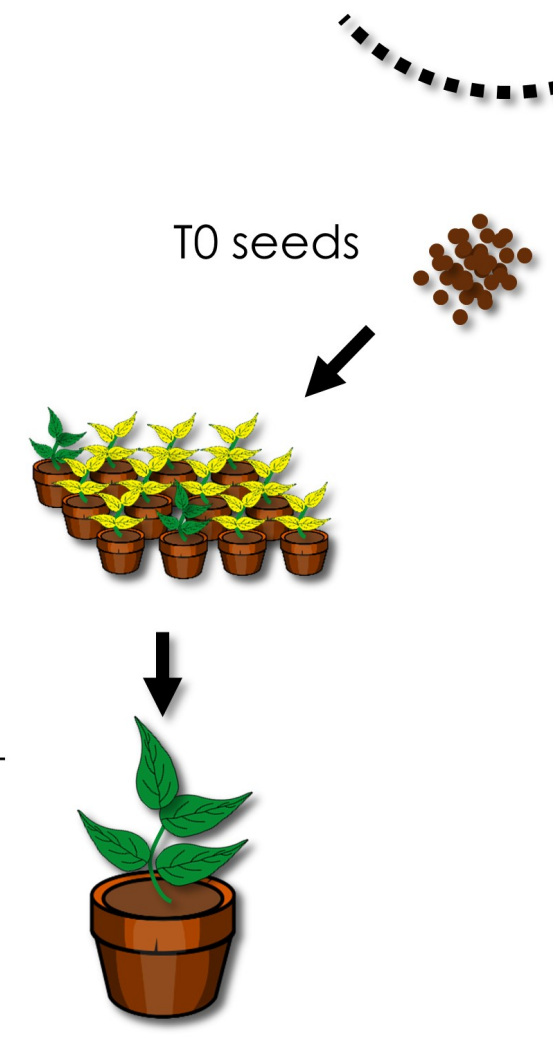

Tissue culturedependent genome engineering

\section{Tissue} culture 

Figure 4:

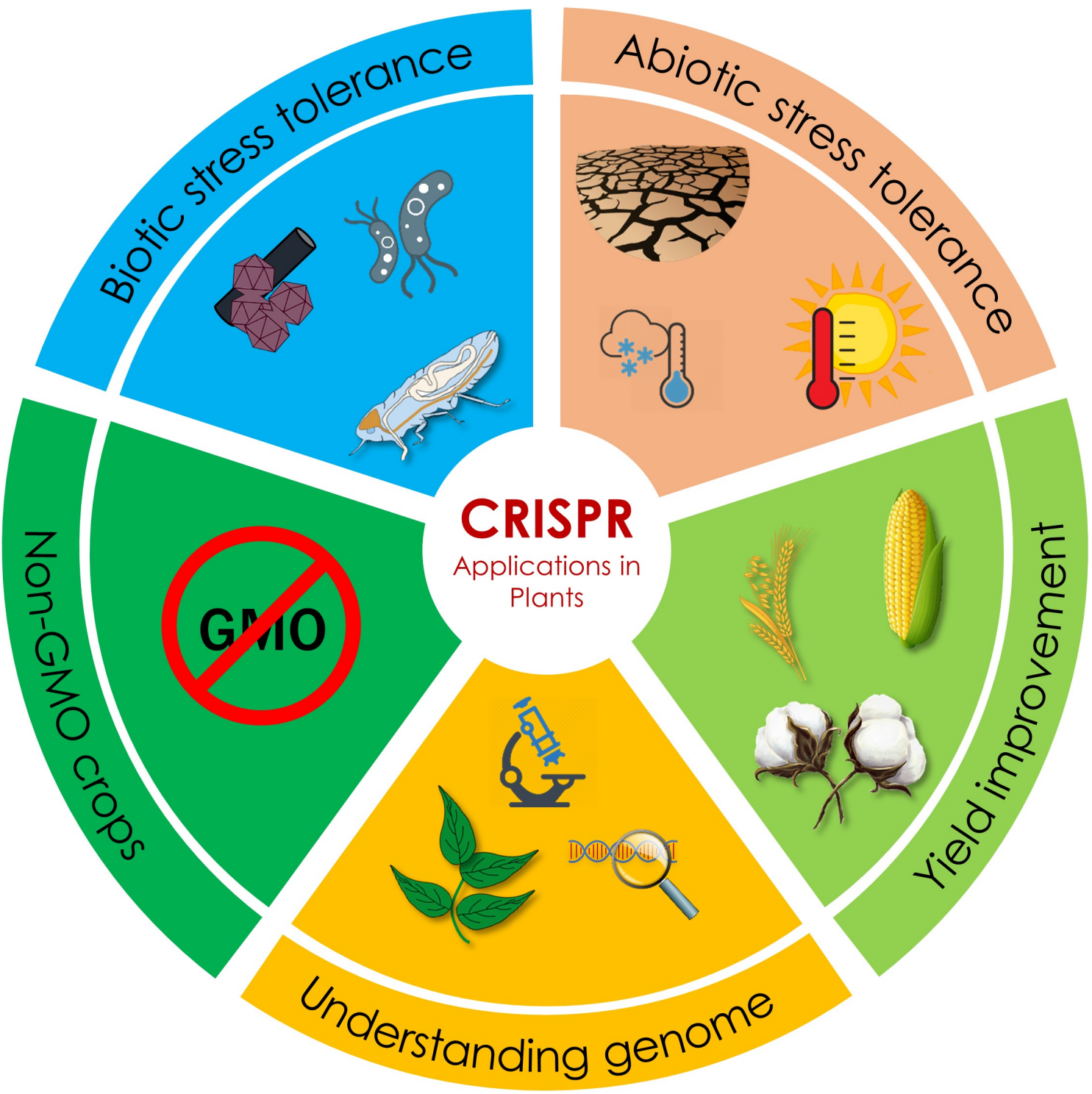

664 